\title{
Influencing factors on antibiotic prescribing in nursing homes
}

Jennifer Russell Principle lecturer, School of Health Care, Oxford Brookes University, and Derek Gallen Director of Postgraduate General Practice Education for the Oxford Deanery, and Primary Care Research Consortium for Northamptonshire

This paper describes a study undertaken to define dimensions of antibiotic prescribing in selected nursing homes and to consider the factors which might have an impact on practice.

Increasing concerns about antibiotic resistance as a result of inappropriate prescribing was the impetus of the study. Poor interprofessional relationships and inadequate GP visiting arrangements were found to impact on prescribing and patient care. Lack of availability of local sensitivity patterns and inadequate use of the laboratory contribute to the problem. The paper draws conclusions from focus group findings and makes recommendations which could improve practice.

Key words: focus groups; interprofessional relationships; methicillin-resistant staphylococcus aureus (MRSA); nursing homes; prescribing

\section{Background}

The purpose of this study was to evaluate antibiotic prescribing in a sample of nursing homes, and to identify factors which might have an impact on practice. Prescribing of all types of medications in nursing homes has been a matter of concern for many years (Spore et al., 1997; Jagger, Bell and Hibbett, 1998; Lunn et al., 1997; Roberts, 1998) in that patients are not only being prescribed inappropriate drugs (Spore et al., 1997) but are also on multiple medications. Studies conducted in nursing homes in the USA found that, on average, each resident was prescribed over seven medications (Broderick, 1997; Lunn et al., 1997). When criteria for appropriate prescribing were developed and applied $54 \%$ of residents were judged to have one or more inappropriate prescriptions. It is against this background and in recognition of increasing concern about antibiotic resistance that antibiotic prescribing needs to be considered. Age associated, physiologic and pathologic changes, functional disability, institutionalization, and invasive devices all

Address for correspondence: Derek Gallen, Primary Care Research Consortium for Northamphtonshire, 3 Queen Street, Wellingborough, North Hants WN8 4RW, UK. Email: dgallen@supanet.com contribute to the high prevalence of infection in nursing home residents (Nicole, Strausbaugh and Garibaldi, 1996; Yoshikawa and Norman, 1996; Medina-Walpole and Katz, 1999), with the most common being: urinary tract infections (cystitis, pyelonephritis), respiratory infections (pneumonia, bronchitis) and skin/soft tissue infections (infected pressure ulcers, celluitis). As a result of this, antimicrobial agent use in nursing homes is intense, and though there is evidence that general practitioners (GPs) have knowledge of the best methods of prescribing (Achterberg, Peerbooms, Bruhl and Tijdschrift voor, 1997; Olesen and Osetergaard, 1995; Peterson et al., 1997; Wood and Abrutyn, 1996; McCleod and Klakovic, 1998) much of it is still done empirically (Nicole, Strausbaugh and Garibaldi, 1996; Vromen et al., 1999; McCleod and Kljakovic, 1998). Even when the laboratory reports there is no infection an antibiotic may still be prescribed (McCleod and Kljakovic, 1998).

Such inappropriate practice, coupled with frequent hospital admissions, has led to antibiotic resistant patterns of gram-negative bacteria in nursing homes (Bonomo and Rice, 1999; Nicole, Strausbaugh and Garibaldi, 1996; McCue, 1997). Escherichia coli is the most common isolate (48\%) followed by proteus spp. (26\%) and other entero- 
bacteriaceae $(20 \%)$. This is a particularly worrying trend when combined with the increase in methicillinresistant staphylococcus aureus (MRSA) (Bradley, 1999; Rao, 1998).

\section{The scale of the MRSA problem}

The colonization of patients with MRSA has been well documented and highlights the scale of the problem in nursing homes (Fraise et al., 1997; Lee, 1996; Lee et al., 1999; Washimo, 1996). One large study (Lee, 1996) found that the presence of colonized patients has been as high as $7.5 \%$ using a combination of screening rectal and nares samples. A percentage of these (5-7\%) go on to develop later infection with MRSA. This study also noted that those in the colonized group were exposed significantly more frequently to antibiotics than the control noncolonized group.

Today's nursing home patients are older, in poorer health, and less able to function independently than has been true in the past (Pell and Williams, 1999). In addition the rotation of nursing homes patients into acute care settings and back again contributes to the increase in colonization with highly resistant bacteria.

\section{Methodology}

Ethical consent was sought and obtained from the local medical ethics committee.

Since this study aimed to examine a range of influential factors on prescribing it was decided following a literature review to employ a focus group methodology. A distinguishing feature of focus group studies is the interaction between participants (Kitzinger, 1999). Focus group methodology is an interviewing technique, not a discussion or a decision making group or a problem solving session (Robinson, 1999). It enables cultural norms to be examined and evaluated in a way individual interviews may not. We decided in the first instance to recruit two groups, one of nurses and one of doctors. Flyers were sent out by using the Health Authority mailing list. These included a description and purpose of the study. We would have liked to employ a sampling technique in selection of group members, but owing to the very small number of applicants we invited everyone who expressed an interest to participate. We recognized from the beginning that such a small response was problematic in validity terms but made a decision to proceed with a view to expanding the study at a later date. We were also concerned that the people who responded would bias out findings by self-selecting from a position of particular interest but the findings indicate that though the majority of the nurses took an interest in staff development, none expressed any interest in prescribing or antibiotic resistance per se. We were struck by the high level of concordance among each professional group; in our discussions there were very few issues over which the nurse of GP group did not reach a consensus.

We attempted to facilitate the group process by inviting the participants to lunch prior to the interview so as to allow us to get to know each other. This was successful in creating a climate of psychological safety to the point that though participants were willing to be acknowledged by name on any future publications, we regarded some of the disclosures to be contrary to the best interests of individuals and organizations represented.

We had prepared a list of issues to be covered by the groups following and in response to our literature review. We used this list to guide the conversation but were careful not to dominate the conversation and as issues of real concern were revealed we sought not to impose our prepared list on the process. We both kept written notes, which were later compared and coded into themes. There was a high level of inter-rater reliability.

We interviewed eight nurses and five doctors though by chance none of the nursing homes were on the registered list of any of the GPs so that thirteen organizations were represented. Most of the nurses were the education lead in their organization, one was employed full time for staff development and one was matron of her organization. All the GPs were full time principals.

It had been our intention to bring both groups together for a subsequent meeting or meetings, but the doctors were unable to clear their diaries to do so.

\section{Data analysis}

Analysis of data was undertaken to identify common convergent themes and also divergent themes. 
To examine the themes within/across the data and also to help verify the emerging analysis, investigator triangulation was undertaken (Flick, 1998). From this analysis the following themes emerged:

\section{Knowledge and awareness of MRSA}

- Knowledge and awareness of MRSA

- Prescribing practices

- Arrangements for GP Visits

- Interprofessional relationships

- Ethical issues

\section{GP focus group}

\section{Knowledge and awareness of MRSA}

The GPs were concerned that their knowledge and awareness of MRSA was generally low and none were aware of the recent clinical guidelines. All felt the Department of Health guidelines to be too long and not helpful. We selected urinary tract infection (UTI) and leg ulcers as markers for prescribing practice and focussed the discussion on these. None of the GP practices represented had a written policy for treating urinary tract infections. All felt that the patients did not need to be visited for an infection unless the nurse believed it to be clinically warranted. When asked about the management of leg ulcers all admitted to a limited knowledge - 'I do not get involved (in this)', 'It's a nursing issue.'

\section{Prescribing practices}

Prescribed antibiotics were usually not related to sensitivity patterns even if the patient was being treated for MRSA colonization or infection. Second and third line antibiotics were commonly prescribed, Cephalexin and Augmentin being the most popular. Both Trimethoprim and Augmentin were in current usage for long-term urinary tract infection prevention. Courses of treatment ranged from three to seven days with three GPs using three day courses, one using a five day course and one a seven day course. The treatment of chest infections was by Augmentin (2 GPs), Cephalexin (1 GP) and Amoxycillin (2 GPs) with courses again ranging from five to seven days, contrary to best practice recommendations. The GPs said that they employ Trimethoprim or Cephotaxime as a first choice for UTIs with a dosage range from three ( $3 \mathrm{GPs})$, to ten days (2 GPs), depending on how ill the patient is perceived to be. All GPs were aware that they did not always prescribe in an evidence-based fashion, citing the pressures they felt from families and nursing staff to do something and their fear of litigation. 'Relatives get upset by a reduction of medication.' 'You may not prescribe but another doctor treating the patient later decides to prescribe and makes you look silly.'

For these reasons they all said that they tended to prescribe prophylactic antibiotics for colds and chest infections. 'If they've got a cold I'll give antibiotics even if I've not bothered to listen to the chest', Amoxycillin being the first line choice. The GPs all felt their knowledge and prescribing patterns had changed in recent years but not as a result of MRSA. In the event of having a patient with MRSA they would follow the advice given by the discharging hospital. The GPs all said that public health concerns did not influence their decision making when prescribing for individual patients but they were aware that lack of information about local sensitivity patterns was impeding good practice. One doctor commented that individual prescribing 'may not be good enough for the population.'

\section{Arrangements for GP visits}

The GPs, none of whom had a regular arrangement to visit local nursing homes, were frustrated by the call-out procedures. They all felt that their relationship with nursing home staff was not good, saying 'They don't provide us with a proper picture of a patients' condition, using descriptions like "gone off". 'They are very disorganised when you visit so it puts you off asking them to get the dressings taken down', 'They ring over trivialities nurse prescribing won't help.' 'They ring at the change of shift because they have to be seen to have done something.'

\section{Inter-professional relationships}

The view of the GPs of nurses was largely negative though they all agreed that the presence of a good nurse manager has a positive influence on the overall care. The GPs were all in agreement with each other that the nurses are 'at the bottom rung 
of their professional ladder and lack professional accountability - they do not understand the time pressures of GPs and do not attempt to make best use of visits.' They believed that nursing homes are very variable in quality and had serious concerns about poor quality care in some instances. 'Would not send my cat' (to stay in certain homes). They felt particularly helpless in influencing the overall quality of life of patients of whom they had no history or knowledge when called out to see as an emergency.

All GPs had concerns about the ethical issues of prescribing, feeling pressurised by nursing staff, relatives and drug companies. 'Pressure from relatives make you practice irrationally for fear of litigation.' The prescribed at times when they knew the population would not be best served by increasing antibiotic usage. The doctors were all in agreement that the current capitation payment of $£ 45$ per year for every patient on their list over 65 years of age irrespective of their place of residence or level of dependency was inadequate and they felt that this may influence the frequency and quality of their visits.

\section{Nurse focus groups}

\section{Knowledge and awareness of MRSA}

The nurses present all had an MRSA policy in place and these policies - though differing in detail - demonstrated an understanding of MRSA and were in line with the National Guidelines (Dar, 1998). They were all clear about the difference between colonization and infection and none had the practice of isolating residents colonised with MRSA. There were different practices in use regarding the management of residents infected with MRSA and some confusion about virulence. Five erroneously believed that MRSA is intrinsically more virulent than methicillin-sensitive staphylococcus aureus (MSSA). Four thought that they could smell MRSA and it had obvious different qualities than MSSA. They all reported they were not afraid to take care of residents with infections and were confident that their practices could prevent spread to themselves or to other patients. Knowledge and training among nurses seemed to be sufficient to provide good care though they acknowledged that they needed to know more. Though they had some misinformation they were applying both national and local policies and were committed to further education. The nurses were all clear and correct about when to suspect a urinary tract infection and when to call the GP to ask for treatment. The nurses reported particular difficulty with knowing when to initiate taking a specimen for laboratory testing. They were concerned that the delay between taking a specimen and the results reaching the GP was likely to endanger the resident and indeed that the specimen may be unusable by the laboratory. Four of the nurses were less confident about how to make a judgement regarding leg ulcer infection.

\section{Prescribing practices}

The nursing group felt that their patients were disadvantaged by the attitude of some GPs 'they regard our patients as second class citizens' though in this case and in others the two nurses whose nursing homes had formal visiting arrangements were less inclined to feel dissatisfied with the service provided for their patients. They reported that most prescriptions were ordered over the "phone without the patient being examined' though the more experienced nurses had no problem with this arrangement if the relationship was generally one of mutual respect. 'Once you get to know each other the trust increases'. Overall the nurses were concerned that prescribing seemed to be haphazard, to vary between GPs and not led by agreed protocols. They felt willing to take responsibility for asking for a prescription but were not confident that prescribing practices were serving their patients well. They were worried that prescribing was a substitute for coming to see the patient. They all reported several patients in each organization on long-term prophylactic antibiotics for the prevention of UTIs.

\section{Arrangements for GP visits}

The nurses reported different arrangements regarding GP visits. Two nursing homes had regular visits of twice or three times per week for which the Practice was paid a fee. All the others depended on call-out arrangement and none of these was satisfactory. The problems ranges from delayed response rate to treating the calling nurse with disrespect or refusing to come out at all. 'It's bad enough trying to talk to the GP but nowadays you have to get past the receptionist before you even get that far.' 
They particularly felt that they were not given sufficient information about patients; 'they don't tell us the whole truth when they want to dump a patient on you especially if the patient is dying'.

Five described methods for making best use of the visit such as faxing the GP with a list of problems.

\section{Inter-professional relationships}

Nurses were eager to tell us they had all particularly chosen this speciality and felt very committed to providing high quality care. They believed that GPs did not respect this fact and regarded them as less than adequate nurses; 'they show lack of trust if they don't know you'; 'they have no respect for our knowledge or judgement'; 'they treat me as if I don't know anything because I am young'.

However those who had established visiting arrangements (two practices) believed that good relationships and mutual respect develop over time. 'It's an individual thing, if they get to know you and trust you they know that you only call out when it is important'.

All felt that GPs undervalued their patient group and often visits were only made on sufferance.

\section{Ethical issues}

Nurses felt compromized by their inability to obtain suitable medical care if their home did not enter into a financial arrangement with the GP practice. They felt they were in a position whereby they needed to defend their patients to obtain best treatment; they felt their own age i.e., the younger nurses, had greater difficulty in arguing for patient care with the doctor. Several felt unable to comply with their professional code of conduct under these circumstances. Nurses felt under the same pressure as GPs from relatives to be seen to be doing something for patients.

\section{Discussion}

\section{Knowledge}

Both professional groups seemed to have sufficient knowledge to provide adequate care for patients colonized or infected with MRSA though knowledge around prescribing best practice was insufficient among GPs. The nurses were adhering to national and local guidelines within their organi- zations but none of the GPs interviewed were adhering to the Department of Health guidelines on MRSA. These findings highlight the need for nurses and GPs to engage in continuing professional development, a need of which nurses were aware.

\section{Prescribing practices}

Antimicrobial agent use in nursing homes has been shown by our study to be empirical rather than based on laboratory results; and this confirms earlier work done in this area (Nicole, Strausbaugh and Garibaldi, 1996; Vromen et al., 1999; McLeod and Kljakovic, 1998). There did seem to be some marked confusion about both the most appropriate first line drug therapies for both urinary track infections and chest infections and for optimum dosage regimens. The nurses highlighted in this study that the prescribing seemed to be haphazard and also felt that prescriptions at times were used as a substitute for the GP making a visit.

Prescribing was affected by the problems of GPs only being called in the acute situation to deal with medical problems in nursing homes and the fact that residents in nursing homes may be registered with several practices. Continuity of care and uniformity of a single practice policy is therefore very difficult to achieve.

\section{Visiting arrangements}

Nursing home residents make greater demands on GPs than they used to in the past and to similar patients living in their own homes (Pell and Williams, 1999). But it was the nature of the demand that caused the most frustration to the GP in our study. They clearly felt that they were called out only to deal with emergencies and not given sufficient or accurate clinical information on which to judge the appropriateness of the call.

This raised the issue of resource allocation. Those GPs who have no contract with a nursing home are expected under the terms of service to provide medical care for elderly residents in nursing homes. Resource transfer has resulted in money being reallocated from the NHS to Local Authorities to facilitate the transfer of patients from long stay beds to the community. This money provides financial support to nursing in residential homes but none of it goes directly to the GP.

The practicalities therefore of GPs looking after an increasing frail and sick members of the com- 
munity who are being transferred out of hospital at an earlier state in their recovery than they previously would have been has placed additionally strain on primary care. No reference is made to the increasing time and resource needed to meet these needs in the NHS Plan (Department of Health, 2001); the Government's response to the Royal Commission on long-term care.

\section{Inter-professional relationships}

There was clearly a strain in the relationship between nursing home staff and GPs in our study group, which is exacerbated by these financial arrangements and by the changing patient population. Both professional groups had a limited respect for and trust in the other and efforts to communicate effectively were limited. We did not find evidence that nursing home owners were attempting to develop ways of addressing these problems.

This issue was the most strongly felt by the participants in this study and surprised us in the depth of feeling.

\section{Ethical issues}

There are clear ethical issues that these two professional groups have identified. The nurses' perception of ageism and second class care being provided to frail elderly people raises important ethical questions. The pressure the doctors feel they are put under to prescribe against their professional judgement may be contributing to an increase in the prevalence of MRSA. There appears to be a mutual intolerance of each other's professional groups, further exacerbating an already unsatisfactory situation in our study group.

The status of frail elderly people in this society is not the remit of this study but it is clearly at the heart of the difficulty.

\section{Conclusions}

Patients are caught in the middle of unsatisfactory arrangements, where care is less than optimal, where there is no evidence of equality of access and where poor interprofessional relationships are impeding quality of care through an extended study would be needed to establish how widespread these are. In addition the risk of increasing the incidence and prevalence of MRSA and other multidrug resistant organisms in the community is enhanced by less than ideal prescribing practices. The revolving door between nursing homes and acute care means that such increase inevitably reaches high risk hospitalized patients.

It is not possible to draw final conclusions from this study alone but its findings in conjunction with those of a large literature review lead us to make the following recommendations and to commence a larger study in the future.

\section{Recommendations}

- Review of GP provision to nursing homes with consideration given to restructuring current reimbursement arrangements;

- Consideration by nursing home owners and managers of the medical supervision needs of their organisations and ways of enhancing interprofessional relationships;

- Intra-professional training and development in the care and management of the elderly;

- Proper and consistent arrangements for the collection of specimens and increased availability of data regarding local antibiotic sensitivities;

- PCG/PCT wide consideration in developing policies and procedures for the provision of GP care to nursing homes.

\section{Acknowledgement}

This work was undertaken by Jennifer Russell and Derek Gallen who received a proportion of it's funding from the NHS Executive: the views expressed in this publication are those of the authors and not necessarily those of the NHS Executive.

\section{References}

Achterberg, W. P., Peerbooms, P. G. and Bruhl, K. G. 1997: Direct antibiotic sensitivity testing in the treatment of urinary tract infections in a nursing home. Gerontologie en Geriatrie 28, 7-10 February 1997.

Bradley, S. F. 1997: Methicillin-resistant Staphylococcus Aureus in nursing homes. Epidemiology, prevention and management. Drugs and Ageing 10, 185-98.

Bradley, S. F. 1999: Issues in the management of resistant bacteria 
in long term care facilities. Infection Control and Hospital Epidemiology 20, 362-6, May 1999.

Bradley, S. F. 1999: Methicillin-resistant Staphylococcus Aureus: long term care concerns. American Journal of Medicine 106, 2s-10s.

Bonomo, R. A. and Rice, L. B. 1999: Emerging issues in antibiotic resistant infections in long term care facilities. Journales of Gerontology Series A, Biological Sciences \& Medical Sciences 54, B260-7, June 1999.

Broderick, E. 1997: Prescribing patterns for nursing home residents in the US. The reality and the vision. Drugs and Ageing 11, 255-6, October 1997.

Dar, R. 1998: PSSRU Survey of Residential and Nursing Home Care. Mental Health Research Review 5, 31-5.

Department of Health. 2001: Primary care, General Practice and the NHS Plan, London: Department of Health.

Flick, U. 1998: An Introduction to Qualitative Research. London: Sage Publications.

Fraise, A. P., Mitchell, K., O'Brien, S. J., Mitchell, K., Oldfield, K. and Wise, R. 1997: Methicillin-resistant Staphylococcus Aureus in nursing homes in a major UK city: An anonymised point prevalence survey. Epidemiology \& Infection 118, 1-5, February 1997.

Jagger, C., Bell, R. I. and Hibbert, H. J. 1998: Residential care for elderly people: Use of medication. Int J Geriatr Psychopharmaco 1, 88-94.

Kitzinger, J. 1999: Introducing Focus Groups in Qualitative Research. Journal of Advanced Nursing 29, 905-13.

Lee, Y. L., Thrupp, L. D., Lee, R., Nothvogel, S., Farsad, N. and Ceasario, T. 1996: Infection Surveillance and antibiotic utilisation in a community-based skilled nursing facility. Ageing (Milano) 8, 113-22, April 1996.

Lee, Y. L. 1996: Colonisation by Staphylococcus Aureus resistant to Methicillin and Ciprofloxacin during 20 months' surveillance in a private skilled nursing facility. Infection Control and Hospital Epidemiology 17, 49-53, October 1996.

Lee, Y. L., Cesario, T., Pax, A., Tran, C., Ghouri, A. and Thrupp, L. D. 1999: Nasal colonisation by Staphylococcus Aureus in active independent, community seniors. Age and Ageing 28, 229-32, March 1999.

Lunn, J., Chan, K., Donoghue, J., Riley, B. and Whalley T. 1997: A study of the appropriateness of prescribing in nursing homes. International Journal of Pharmacy Practice 5, 6-10.

McCue, J. D. 1997: Antibiotic resistance: Why is it increasing in nursing homes? Geriatrics 52, 34-6, 39-43, July 1997.

Mcleod, D. and Kljakovic, M. 1998: What do general practitioners do when patients present with symptoms indicative of urinary tract infections? New Zealand Medical Journal 111, 189-91, May 1998.

Medina-Walpole, A. M. and Katz, P. R. 1999: Nursing home acquired pneumonia. Journal of American Gerontology Society. 47, 1005-15, August 1999.

Monnet, D. L. 1998: Methicillin-resistant Staphylococcus Aureus and its relationship to Antimicrobial use: possible implications for control of Infection. Control \& Hospital Epidemiology 19, 552-9, August 1998.

Namnyak, S. 1998: Methicillin-resistant Staphylococcus Aureus: a questionnaire and microbiological survey of nursing and residential homes in Barking, Havering and Brentwood. Journal of infection 36, 67-72, January 1998.

Nicole, L. E., Strausbaugh, L. J. and Garibaldi, R. A. 1996: Infections and antibiotic resistance in nursing homes. Clinical Microbiology Reviews 9, 1-17, January 1996.

Olesen, F. and Osetergaard, I. 1995: Patients with urinary tract infection: proposed management strategies of general practitioners, microbiologists and urologists. British Journal of General Practice , 45, 611-3 November 1995.

Pell, J. and Williams, S. 1999: Do Nursing Home Residents Make Greater Demands on GPs? A Prospective Comparative Study. British Journal of General Practice 49, 527-30.

Peterson, G. M., Stanton, L. A., Bergin, J. K. and Chapman, G. A. 1997: Improving the prescribing of antibiotics for urinary tract infections. Journal of Clinical Pharmarcology and Therapeutics 22, 147-53, April 1997.

Rao, G. G. 1998: Risk factors for the spread of antibiotic-resistant bacteria. Drugs 55, 323-30, March 1998.

Roberts, M. S. 1998: Medication prescribing and administration in nursing homes. Age and Ageing 27, 385-92.

Robinson, N. 1999: The use of Focus Group Methodology with Selected Examples from Sexual Health Research. Journal of Advanced Nursing 29, 905-13.

Spore, D. L., Mor, V., Larrat, P., Hawes, C. and Hiris, J. 1997: Inappropriate drug prescriptions for elderly residents of board and care facilities. American Journal of Public Health, 87, 404-9, March 1997.

Taylor, L. 1997: MRSA. Nursing Standard 11, 1-19, August 1997.

Yoshikawa, T. T. and Norman, D. C. 1996: Approach to fever and infections in nursing homes. Journal of American Gerontology Society 44, 74-82, January 1996.

Vromen, M., Van Der Ven, A. J., Knols, A. and Stobberingh, E. E. 1999: Antimicrobial resistance patterns in urinary isolates from nursing home residents. Fifteen years of data reviewed. Journal of Antimicrobial Chemotherapy 44, 113-6, July 1999.

Washio, M. 1996: Incidence of Methicillin-resistant Staphylococcus Aureus isolation in a skilled nursing home: a third report on the risk factors for the occurrence of MRSA infection in the elderly. Journal of Epidemiology 6, 69-73, June 1996.

Wood, C. A. and Abrutyn, E. 1996: Optimal treatment of urinary tract infections in elderly patients. Drugs and Ageing 9, 35262, November 1996. 